\title{
Passivity of Interconnected Asynchronous Discrete-Time Systems
}

\author{
J.R. Cueli* C. Canudas-de-Wit ${ }^{* *}$ \\ * INRIA Rhône-Alpes \\ Inovallée, 655 avenue de l'Europe, \\ Montbonnot 38 334, Saint Ismier Cedex, France \\ jose.ramos-cueli@inrialpes.fr \\ ** CNRS, GIPSA-Lab, Grenoble, France \\ carlos.canudas-de-wit@gipsa-lab.inpg.fr
}

NeCS project-team

\begin{abstract}
This paper presents a constructive algorithm to design local controllers for feedback systems that are interconnected via time-varying and asynchronous sampling. These systems result in many application fields such as remotely-operated systems, interconnected vehicle control loops, and more generally in component-based control design where synchronous exchange of information is not feasible. The design is based on the (MASP) MAximum Sampling time preserving Passivity, and uses discrete-time passivity considerations. The paper first explores several ways to compute the MASP for linear systems, and then proposes a numerical algorithm to compute local feedback loops providing a MASP compatible with the maximum sampling-time upperbound of each sub-system. This results in a exponentially stable interconnection. The paper also presents a simulation example of this design.
\end{abstract}

Keywords: Passive systems, Discrete-time systems, VSP

\section{INTRODUCTION}

This paper is devoted to the study of passivity properties of asynchronously non-uniformly sampled systems. The idea of studying these systems comes from the necessity of developing theoretical tools for the analysis of networked and embedded control systems, which usually operate under variable resources like communication rates and computational loads. A similar need also comes from the intention of interconnect block systems via asynchronous exchange of information. In these circumstances, the sampling time used for each of the subsystems is no longer a fixed parameter, and it may be adapted on the fly as a function of the available resources at the moment. Hopefully, this strategy would achieve a reduction of the costs (energy and/or computation), although it obviously depends on the application at hand.

Passivity-based control is a well established method (see for instance Brogliato et al. [2007]) that has been used in a variety of applications such as: transmission lines, (compressible) fluid pipelines, mechanical resonances in electromechanical systems, distributed elasticity, avionics, etc. The use of the passivity ideas in the context described above requires a knowledge of the conditions under which the passivity of these systems is preserved under variable sampling time. Although, passivity lost does not always

\footnotetext{
* The first authors would like to acknowledge the INRIA for the financial support provided during his Post-Doctoral studies at Grenoble in the research NeCS team. The second author gratefully acknowledge the suggestions of Prof. M. López.
}

imply lost of stability, the adaptation of the passivity design principles to asynchronous interconnected systems can be seen as step forward to design controllers for embedded event-driven systems. To some extent the line of research are related to the notion of any-time stability ideas mentioned in Murray et al. [2003], and to studies on asynchronous event-driven systems like the ones in Rantzer and Byrnes [2003], Robert [2007], Nilsson and Bernhardsson [1997], Marti et al. [2002]. The work reported in Hassibi et al. [1999] has extended the Lyapunov stability theorem for asynchronous systems with the notion of Lyapunov function monotonically decreasing in average. In Laila et al. [2001], the authors study conditions under which the discretization of a continuous-time nonlinear system satisfying a certain dissipation inequality, yields a discretetime system with similar dissipation properties (in a semiglobal practical sense).

In practice, it can be assumed that the sampling time, $T$, belongs to some interval, $T \in\left(0, T_{\max }\right]$, and a controller should be designed to robustly stabilize the system for any possible value of the sampling period in that interval. Examples can be found in tele-operated systems under variable time-delay transmission where passivity enables the reliable and safe control of such systems, see Hokayem and Spong [2006] and references therein. Another example can be found in CAN buses in the automotive industry where many feedback subsystems interact in a non synchronous manner. 
In this paper we deal with the following issues. First we introduce the notion of (MASP) MAximum Sampling time preserving Passivity for linear systems; given a continuoustime system with some dissipation properties specified, the notion of MASP give a maximum sampling time, $T^{*}$ after which passivity is lost. This type of result is similar to the one in Laila et al. [2001] for nonlinear systems. Because in this paper we deal with linear systems, it is then possible to devise a numerical algorithm for the exact computation of $T^{*}$. Another interesting result along these lines is Rohrer and Nosrati [1981], where given a passive sampled $R L C$ circuit, the maximum sampling time at a given instant preserving passivity has been found to be proportional to dissipation/stored energy ratio of the circuit. The conclusion (similar to one obtained here) is that if a passive circuit without energy dissipation elements is discretized, it losses passivity whatever the sampling time is.

A second aspect studied here concerns the case of system locally asynchronous but globally synchronous feedback interconnected systems. The notion of globally synchronous comes from the fact that we limit this study to samples $T_{i}$ of each $i$-subsystem that are multiple integers among them, nevertheless we allows the sampling time of each individual sub-systems to be time-varying. Finally, we use these results as a design guidelines for the control design, and we propose a numerical algorithm to compute local feedback loops providing a MASP compatible with the maximum sampling-time upperbound of each subsystem. These results in a exponentially stable interconnection. The paper also presents a simulation example of this design.

\section{PROBLEM FORMULATION}

Consider linear system of the form

$$
\left\{\begin{array}{l}
\dot{x}(t)=A_{c} x(t)+B_{c} u(t) \\
y(t)=C x(t)+D u(t)
\end{array}\right.
$$

We assume that the above continuous-time system belong to a general class of dissipative system, that is, it satisfies the dissipation inequality Márquez [2003], Brogliato et al. [2007]

$$
\int_{0}^{t} u^{T} y d t \geq V(t)-V(0)+\int_{0}^{t} w(u, y, x) d t \geq-\beta
$$

where $w(u, y, x)$ is the dissipation rate, and $V(t) \geq 0$ is the energy storage function, which vanishes at the equilibrium point. According to the particular form of $w(u, y)$, systems can be classified as passive, strictly passive, (input, or output) passive, etc. For instance if the dissipation rate given by

$$
w(u, y, x)=q y^{T} y+r u^{T} u+\rho V(x)
$$

with $r, q, \rho>0$, and $V(x)$ is a positive definite scalar function, the system will be classified as strictly input/output/state passive system. As it will be seen latter, strict passivity plays an important role here in connection with the existence of MASP. $Q S R$ dissipatedness is a generalization of the concept of passivity for quadratic dissipation supply functions of the form:

$$
\begin{aligned}
w(u, y, x) & =y^{T} Q y+2 y^{T} S u+u^{T} R u+\rho V(x)= \\
& =\left(\begin{array}{ll}
y^{T} & u^{T}
\end{array}\right)\left(\begin{array}{cc}
Q & S \\
S^{T} & R
\end{array}\right)\left(\begin{array}{l}
y \\
u
\end{array}\right)+\rho V(x)
\end{aligned}
$$

For linear systems as the one consider here, the storage function $V(x)$ can be assumed to be quadratic, i.e. $V(x)=$ $x^{T} P x$. With regard to the problem considered in this paper, we assume that the continuous-time system (1) fulfill the following strict passivity property:

Property 1. There exist matrices $P=P^{T}>0, Q>0, R>$ $0, S$ and a scalar $\rho>0$ such that the following inequality holds:

$$
\underbrace{\left(\begin{array}{l}
A_{c}^{T} P+P A_{c} P B_{c}-C^{T} \\
B_{c}^{T} P-C-\left(D+D^{T}\right)
\end{array}\right)}_{\triangleq W} \leq-M<0
$$

with $M \triangleq\left(\begin{array}{ll}C^{T} Q C+\rho P & C^{T} Q D+C^{T} S \\ D^{T} Q C+S^{T} C & D^{T} Q D+S^{T} D+D^{T} S+R\end{array}\right)$

If this hypothesis hold with $R=Q=\rho=0$, the system is passive (not strictly), and the transfer function is positive real (not strictly).

Note also that this property implies, among other things that: i) $A_{c}$ should be strictly stable, and in particular that ii) $\left(D+D^{T}\right)>0$. Indeed the presence of a direct term $D$ is a necessity for a system to preserve passivity after discretization. This has already been reported previously Brogliato et al. [2007].

To simplify further the discussion here we assume that the matrix at the right hand side of (5), matrix $M$, is upper bounded by the matrix $-\epsilon I$, which in turn implies that

$$
W \leq-\epsilon I<0, \text { with } \epsilon \triangleq \lambda_{\min }\{M\}>0 .
$$

the scalar $\epsilon$, describes the level of dissipatedness that the continuous-time system is assumed to possess. This quantity will be related to the maximum sampling time tolerated before lost of passivity of the discrete-time representation.

\subsection{Dissipation in discrete-time systems}

The discrete time representation of the continuous system presented before is given by

$$
\begin{aligned}
x_{k+1} & =A_{k} x_{k}+B_{k} u_{k} \\
y_{k} & =C x_{k}+D u_{k}
\end{aligned}
$$

where

$$
\begin{aligned}
A_{k}=A\left(T_{k}\right) & =e^{A_{c} T_{k}} \simeq I+A_{c} T_{k}+\frac{A_{c}^{2} T_{k}^{2}}{2 !}+\frac{A_{c}^{3} T_{k}^{3}}{3 !}+\cdots \\
B_{k}=B\left(T_{k}\right) & =A_{c}^{-1}\left(e^{A_{c} T_{k}}-I\right) B_{c}=\left(e^{A_{c} T_{k}}-I\right) A_{c}^{-1} B_{c} \\
& \simeq\left(I+\frac{A_{c} T_{k}}{2 !}+\frac{A_{c}^{2} T_{k}^{2}}{3 !}+\cdots\right) B_{c} T_{k}
\end{aligned}
$$

and $A_{c}$ is Hurwitz. Note that these expressions are welldefined (analytical) if the sampling is regular (in the sense defined in Araki [1993]) even when $A_{c}$ is non invertible, since the inverse of $A_{c}$ does not appear in the expansion of $B_{k}$ Robert [2007]. In addition, this regularity condition is also needed for preserving controllability and observability of the discretized system. In particular, the sampling time should not be multiple of the difference between the imaginary part of two eigenvalues, as shown in Araki [1993]. 
Finally, remark that exact discretization preserves stability for any sampling time, but not necessarily maintain its original passivity properties (if any).

The dissipation rate and energy storage functions definitions are similar both for continuous or discrete time systems. The variation of the storage function cannot be greater, by definition, than the energy supplied to the system in a given interval of time. That is:

$$
\sum_{i=0}^{k-1} T_{i} u_{i}^{T} y_{i} \geq V_{k}-V_{0}+\sum_{i=0}^{k-1} T_{i} \varpi\left(u_{i}, y_{i}, x_{i}\right)
$$

for some $V_{k}=x_{k}^{T} P x_{k}$, and some $P=P^{T}>0$. Function $\varpi$ may have a similar form than the one for the continuoustime case. Note that this is a better adapted dissipation definition for the case of non-uniform sampled systems.

\subsection{Passivity relations between the continuous and the} discrete-time case

Let $\xi(t)=\left[x(t)^{T}, u(t)^{T}\right]^{T}$, and $\xi_{k}=\left[x_{k}^{T}, u_{k}^{T}\right]^{T}$. The dissipation inequality $(2)$ can be written as

$$
\dot{V}(x)-u^{T} y \triangleq \xi^{T}(t) W \xi(t) \leq-\xi^{T}(t) M \xi(t) \leq-\epsilon\|\xi(t)\|^{2}
$$

where $w$ has been rewritten only as a function of $x$, and $u$, by substitution of $y=C x+D u$, i.e.

$$
w(u, y, x)=w(u, x)=\xi^{T}(t) M \xi(t)
$$

with the matrix $M$ as defined in Property 1 . Note that (11) can be also rewritten as:

$$
\xi^{T}(t)[W+M] \xi(t) \leq \xi^{T}(t)[W+\epsilon I] \xi(t) \leq 0
$$

with $W<0, M>0$, and $\epsilon>0$ as defined before. Introducing this in (11) and taking samples at $t=t_{k}$, gives:

$$
x_{k+1}^{T} \frac{P}{T_{k}} x_{k+1}-x_{k}^{T} \frac{P}{T_{k}} x_{k}-u_{k}^{T} y_{k} \leq-\xi_{k}^{T} M \xi_{k} \leq-\epsilon\left\|\xi_{k}\right\|^{2}
$$

Regrouping the terms at the left hand side of the inequality and combining this with system (7), we get

$x_{k+1}^{T} \frac{P}{T_{k}} x_{k+1}-x_{k}^{T} \frac{P}{T_{k}} x_{k}-u_{k}^{T} y_{k} \triangleq \xi_{k}^{T} W_{d}\left(T_{k}\right) \xi_{k} \leq-\xi_{k}^{T} M \xi_{k}$

or,

with,

$$
\xi_{k}^{T}\left[W_{d}\left(T_{k}\right)+M\right] \xi_{k} \leq \xi_{k}^{T}\left[W_{d}\left(T_{k}\right)+\epsilon I\right] \xi_{k}
$$

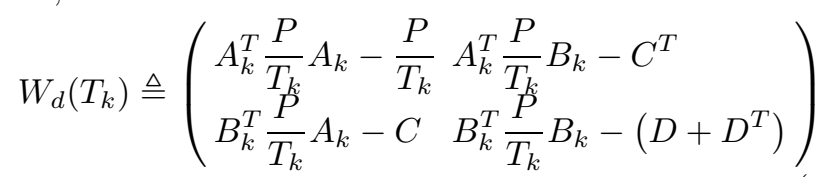

Note that as long as $W_{d}\left(T_{k}\right)+\epsilon I \leq 0$, then the discretetime dissipation inequality (10) holds with $\varpi_{k} \triangleq \xi_{k}^{T} M \xi_{k}$.

Summarizing we have the following two cases:

i) $\xi^{T}(t)[W+\epsilon I] \xi(t) \leq 0$ for the continuous-time case, and

ii) $\xi_{k}^{T}\left[W_{d}\left(T_{k}\right)+\epsilon I\right] \xi_{k} \leq 0, \forall T_{k} \in\left(0, T^{*}\right]$ for the discretetime case.

The case $i$ ) results form the inherent dissipation property of the continuous-time system, whereas the discrete-time property $i i$ ) need to be verified by finding a sampling time
$T^{*}$. The value, $T^{*}$, is named here MAximum Sampling time preserving Passivity; MASP. Conditions for the simple case of passivity $\left(\varpi_{k}=0 \Rightarrow \epsilon=0\right)$ are well known and given by the KYP lemma for discrete time systems, see for instance Navarro-López [2002], or Navarro-Lopez [2005]. It states that if there exist $P=P^{T}>0$ such that the matrix $W_{d}\left(T_{k}\right)$ is negative semidefinite, then, the discrete-time system (7) is passive. Robust control problems require an extension of that lemma, like the parametric KYP lemma in El-Ghaoui and Niculescu [2000].Note that here we will consider a more general case where $\varpi_{k} \neq 0$. As mentioned before our objective here is to characterize the larger $T_{k}$, namely $T^{*}$, such that $W_{d}\left(T_{k}\right)+\epsilon I \leq 0, \forall T_{k} \leq T^{*}$. The following section provide such results and next two subsequent sections deals with the problem of interconnections, and control design, respectively.

\section{PASSIVITY OF NON-UNIFORM SAMPLED OPEN-LOOP LINEAR SYSTEMS}

In this section we present some results concerning nonuniform sampled open-loop systems. We first seek to demonstrate that, under the dissipation hypothesis of the continuous-time system, there always exists a MASP, and that it can be easily computed numerically. We then present some approximations for the computation of $T^{*}$ in closed form.

Theorem 2. Maximum sampling time preserving passivity. Assume that the plant (1) is dissipative in the sense of (6) for some $\varepsilon>0$, that is, it satisfies the dissipative inequality $W \leq-\varepsilon I$. Then there exist $T^{*}>0$ given as

$$
T^{*} \triangleq \inf _{0<T_{k}<T_{M}}\left\{\operatorname{det}\left(W_{d}\left(T_{k}\right)+\epsilon I\right) \equiv 0\right\}
$$

with

$$
T_{M} \triangleq \frac{\operatorname{det}\left(D+D^{T}\right)}{\operatorname{det}\left(C P_{c}^{-1} C^{T}\right)}
$$

such that the exact discrete-time system (7) is passive $\left(W_{d}\left(T_{k}\right) \leq 0\right)$, for all $T_{k} \in\left(0, T^{*}\right]$.

Remark $T_{M}$ is always defined if the dynamical system is a minimal realization system such that $C$ is full rank.

Proof We first show that there exist a computable upper bound on $T^{*} \leq T_{M}$, with $T_{M} \triangleq \frac{\operatorname{det}\left(D+D^{T}\right)}{\operatorname{det}\left(C P_{c}^{-1} C^{T}\right)}$. Secondly, it is trivial to see that by definition $\lim _{T_{k} \rightarrow 0^{+}}\left\{W_{d}\left(T_{k}\right)\right\}=$ $W \leq-\epsilon I$, and that $W_{d}\left(T_{k}\right)$ is continuous in $T_{k}$. Hence, if a $T_{M}>0$ do exist, then by continuity $W_{d}\left(T_{k}\right)$ should become non negative definite for some finite $T^{*}<T_{M}$.

To complete the first statement (the other two are trivial), note that inequality (13) may be rewritten as

$$
\begin{aligned}
W_{d}\left(T_{k}\right)= & -\underbrace{\left(\begin{array}{ll}
P_{c} / T_{k} & C^{T} \\
C & D+D^{T}
\end{array}\right)}_{\mathcal{C}}+ \\
& +\underbrace{\left(A\left(T_{k}\right) B\left(T_{k}\right)\right)^{T} \frac{P_{c}}{T_{k}}\left(A\left(T_{k}\right) B\left(T_{k}\right)\right)}_{\mathcal{A}}
\end{aligned}
$$

with $\mathcal{A}=\mathcal{A}^{T} \geq 0$ and $\mathcal{C}=\mathcal{C}^{T}$. A sufficient condition for losing passivity, that is $(-\mathcal{C}+\mathcal{A}+\epsilon I)>0$, is that $T_{k}$ be large enough to make $\mathcal{C}<0$. 
Since the continuous time system is strictly input/state passive, $D+D^{T}>0, P_{c}>0$. Schur complement and the properties of the determinants (see Corollary 18.1.7. of Harville [1997]) are sequentially applied to conclude that

$$
\begin{gathered}
\mathcal{C} \leq 0 \Longrightarrow D+D^{T}-T_{k} C P_{c}^{-1} C^{T} \leq 0 \Longrightarrow \\
\Longrightarrow \operatorname{det}\left(D+D^{T}\right) \leq T_{k} \operatorname{det}\left(C P_{c}^{-1} C^{T}\right)
\end{gathered}
$$

Thus any $T_{k}>T_{M}$, with

$$
T_{M} \triangleq \frac{\operatorname{det}\left(D+D^{T}\right)}{\operatorname{det}\left(C P_{c}^{-1} C^{T}\right)}
$$

does not result in a negative definite $W_{d}\left(T_{k}\right)$. Note that the value of $T_{M}$ depends indirectly on the plant dynamics $A_{c}$ and $B_{c}$, through its energy storage function matrix $P_{c}$.

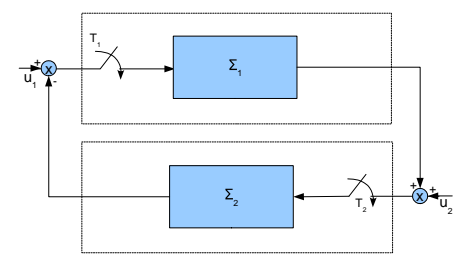

Fig. 1. Feedback passive discrete time system

\section{PASSIVITY OF ASYNCHRONOUSLY INTERCONNECTED SYSTEMS}

In this section, we consider the case of two (open-loop) passive continuous-time systems, $\Sigma_{i}$, interconnected in feedback, and asynchronously sampled as shown in Figure 1 . We assume that the input of system $\Sigma_{1}$ comes from a zero order hold $(\mathrm{ZOH})$ with sampling time $T_{1, k}$, whereas its output is sampled and hold with a period equal to $T_{2, k}$. Both $T_{1, k}$ and $T_{2, k}$ are allowed to be different and are time-varying (non-uniform sampling). As a consequence the interconnected system is asynchronous. In this paper we limited to the case where $T_{2, k}=n_{k} T_{1, k}$, where $n_{k} \in\left\{1,2, \ldots, n_{\max }\right\}$.

Following the previous section analysis, we are interested in characterizing how each of the MASP and the passivity properties of each subsystem are related for this interconnected setup. These results will be used in the subsequent section where control design will be presented.

Lemma 1. Assume that exist, $T_{1}^{*}, T_{2}^{*}$, verifying $T_{i, k} \leq$ $T_{i}^{*}$, such that each sub-system $\Sigma_{i}$ fulfill its respective dissipation inequality (10), with $\varpi_{l}=\xi_{l}^{T} M_{l} \xi_{l} \leq \epsilon_{l}\left\|\xi_{l}\right\|^{2}$, for $l=1,2$. Then the feedback interconnection of the two systems gives an exponentially stable closed-loop system.

Proof Sampling times are assumed to be multiple integer $T_{2, k}=n_{k} T_{1, k}$. To simplify notation we use $T_{1, k}=T_{k}$, and $T_{2, k}=n T_{k}$. As by hypothesis we also assume that each subsystem $\Sigma_{i}$ is dissipative in the sense of the equation (10), and hence its discretized version satisfy the following inequalities for the first sub-system $\left(\Sigma_{1}:-u_{k} \mapsto y_{k}\right)$ :

$$
-\sum_{i=0}^{k-1} T_{i} y_{i}^{T} u_{i} \geq V_{1, k}-V_{1,0}+\sum_{i=0}^{k-1} T_{i} \varpi_{1}\left(y_{i},-u_{i}, x_{1, i}\right)
$$

and for the second sub-system $\left(\Sigma_{2}: y_{n k} \mapsto u_{n k}\right)$ :

$$
\sum_{i=0}^{k-1} n_{i} T_{i} y_{n i}^{T} u_{n i} \geq V_{2, n k}-V_{2,0}+\sum_{i=0}^{k-1} n_{i} T_{i} \varpi_{2}\left(y_{n i}, u_{n i}, x_{2, n i}\right)
$$

As by hypothesis the second system is under sampled $\left(n_{i} \geq 1\right)$, then inequality (19), also holds for samples smaller than $n_{k} T_{k}$, and in particular for $T_{k}$. Hence it also applies that, for $\Sigma_{2}^{\prime}: y_{k} \mapsto u_{k}$ :

$$
\sum_{i=0}^{k-1} T_{i} y_{i}^{T} u_{i} \geq V_{2, k}-V_{2,0}+\sum_{i=0}^{k-1} T_{i} \varpi_{2}\left(y_{i}, u_{i}, x_{2, i}\right)
$$

Adding (18) and (20), gives

$$
\begin{aligned}
0= & \sum_{i=0}^{k-1} T_{i} y_{i}^{T} u_{i}-\sum_{i=0}^{k-1} T_{i} y_{i}^{T} u_{i} \geq V_{1, k}-V_{1,0}+V_{2, k}-V_{2,0}+ \\
& +\sum_{i=0}^{k-1} T_{i} \varpi_{1}\left(y_{i},-u_{i}, x_{1, k}\right)+\sum_{i=0}^{k-1} T_{i} \varpi_{2}\left(y_{i}, u_{i}, x_{2, i}\right)
\end{aligned}
$$

Define $V_{k} \triangleq V_{1, k}+V_{2, k}$. We have

$$
\begin{aligned}
V_{k} & -V_{0} \leq-\sum_{i=0}^{k-1} T_{i} \varpi_{1}\left(y_{i},-u_{i}, x_{1, i}\right)-\sum_{i=0}^{k-1} T_{i} \varpi_{2}\left(y_{i}, u_{i}, x_{2, i}\right) \\
& \leq-\bar{T} \sum_{i=0}^{k-1}\left[\varpi_{1}\left(y_{i},-u_{i}, x_{1, i}\right)+\varpi_{2}\left(y_{i}, u_{i}, x_{2, i}\right)\right]<0
\end{aligned}
$$

where $\bar{T}=\min _{k \geq 0}\left\{T_{k}\right\}$. From this equation we get

$$
\begin{aligned}
V_{k+1}-V_{k} & \leq-\bar{T}\left[\varpi_{1}\left(y_{k},-u_{k}, x_{1, k}\right)+\varpi_{2}\left(y_{k}, u_{k}, x_{2, k}\right)\right] \\
& \leq \bar{T}\left[\epsilon_{1}+\epsilon_{2}\right]\left\|\xi_{k}\right\|^{2}<0
\end{aligned}
$$

This results from the fact that $\varpi_{l}=\xi_{l}^{T} M_{l} \xi_{l} \leq \epsilon_{l}\left\|\xi_{l}\right\|^{2}$, for $l=1,2$, with $\xi_{k}^{T}=\left[\xi_{1, k}^{T}, \xi_{2, k}^{T}\right]$. Hence $V_{k}$ is a Lyapunov function of the closed loop interconnection, which is exponentially stable since the system is strictly state passive.

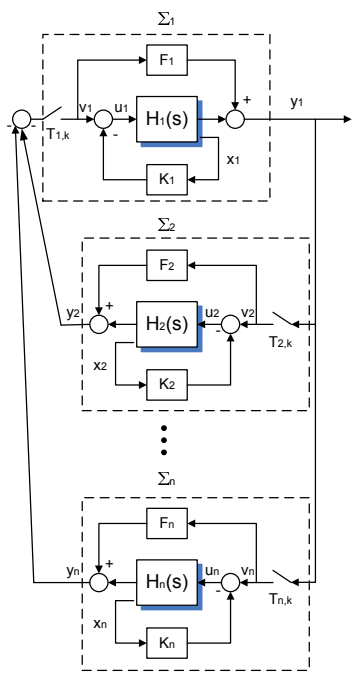

Fig. 2. Feedback interconnections

\section{CONTROL DESIGN}

The control scenario considered in this section is shown in Figure 2. This scenario consider a set of feedback interconnected systems exchanging information in asynchronously manner with different, and possible time-varying, sampling periods $T_{i, k}$. These scenarios can be found in several applications. For instance, remotely-operated systems based on 
passivity arguments is a natural domain for application of these ideas. Other potential field is in vehicle control where many control subsystems (ABS, ESP, TCS, ECU, etc.) are feedback interconnected and exchange information via the CAN bus. More generally, applications based in component-oriented design without having the possibility to operate synchronously, are also a home-land for this type of design.

As in many of these applications, we can not expect that each of the open-loop systems, $H_{i}(s)$, already possed the desired dissipation properties, we may assume that the designer have the possibility to design local feedback loops, (i.e. matrices $K_{i}$, and $F_{i}$ in Fig. 2), with the objective to impart the desired dissipation properties to the resulting discrete-time system. In particular, it is suited that after closing the local feedback loops, each controlled subsystem results with a MASP consistent with the domain of variation of each sampler. As a result, the systems can be feedback interconnected with another system yielding stable behaviour under this asynchronous sampling interconnection.

Assumptions 3. With regard to Figure 2, we consider the following assumptions (to simplify notation we do not explicitly notate the subsystem index, $i$, unless needed):

i) Each system is locally feedback controlled by control structure shown in Fig. 2.

ii) Each controlled feedback system results in

$$
\left\{\begin{array}{l}
\dot{x}(t)=\left(A_{c}-B_{c} K\right) x(t)+B_{c} v(t) \\
y(t)=C x(t)+(D+F) v(t)
\end{array}\right.
$$

with $\left(A_{c}, B_{c}\right)$ stabilizable pair.

iii) The input signals of each controlled subsystem, $v(t)$ are sampled and hold with a sampling time $T_{k}$ which is assumed to vary between two known bounds; $T_{k} \in$ $\left[T^{m}, T^{M}\right]$.

Conversely to the problem studied in the previous section where the dissipative properties of each subsystem where given, here the problem is to design each of the matrices $K$, and $F$ such as the resulting discrete system with the time basis $T_{k}$, has a MASP $T^{*}>T^{M}$.

Design control algorithm. Assume that a minimal realization of the plant is given. The algorithm seeks to produce a local state feedback $(K)$ and feedforward $(F)$ such that the MASP is greater than $T^{M}$. This algorithm is heuristic and it works by increasing the level of dissipatedness of the local feedback system in each step by a factor of $\Delta$, where $\Delta$ is a small positive number. The algorithm follows the next steps:

(1) Initialize $\epsilon=\Delta$.

(2) Compute $K, F$ such that the system is passive with a minimum level of dissipativity ${ }^{1} \epsilon$ by solving the following LMI

$$
\left(\begin{array}{lr}
X A_{c}^{T}+A_{c} X-Z B_{c}^{T}-B_{c} Z, & B_{c}-X C^{T} \\
B_{c}^{T}-C X, & -D^{\prime}-D^{\prime T}
\end{array}\right) \leq-\epsilon I
$$

where $D^{\prime}=D+F$ and the unknowns are $X=$ $P^{-1}, Z=P^{-1} K$ and $F$. This inequality results

\footnotetext{
1 Note that $\epsilon$ here does not follow the definition (6) It is only used as a mean to quantify the amount of dissipativity imparted by the design control gain
}

form the change of coordinates $(z=P x)$, and the new factorization in terms of $(z, v)$ of the expression $\dot{V}\left(P^{-1} z\right)-v^{T} y$.

The feedback gain is obtained from the relation $K=Z P$. If the problem is non feasible, the chosen value of $\Delta$ was too high or either the problem has not any solution.

(3) Compute the MASP $T^{*}$ of the system. The exact solution is determined by the first zero crossing of $\operatorname{det}\left[W_{d}(T)\right]$.

(4) If $T^{*} \geq T^{M}$, the problem has been solved. If not, then increase $\epsilon=\epsilon+\Delta$, and back to the step 2 .

Now if this algorithm is successfully applied to each subsystem $\left(T_{i}^{*} \geq T_{i}^{M}\right)$, and if the sampling times are multiple integer among them, then in virtue to the Lemma (1), the interconnection of several sub-systems as indicated in Fig. 2 will result in a exponentially stable closed-loop.

\subsection{Example.}

Consider the system shown in Figure 2, with the open-loop system $H_{1}(s)=\frac{1}{S}$ being a simple integrator. For simplicity of the presentation we assume that the system $H_{2}(s)$ has been already stabilized resulting in a closed-loop system $\Sigma_{2}(s)$ given by

$$
\Sigma_{2}(s)=\frac{-0.0376 s^{2}+0.0896 s-0.0533}{s^{4}+2.9737 s^{3}+2.8217 s^{2}+0.9142 s+0.0908}+1
$$

We assume that the sampling times are different for each block: $T_{1}=1 \mathrm{sec}$. and $T_{2}=2 \mathrm{sec}$.

The problem is to design $k_{1}$ and $f_{1}$, such that the closed loop system remains stable. This can be done using the numerical algorithm proposed before, but as the example is simple enough the controller parameters can be designed in closed form, as a function of the approximated value of $T^{*}$. Numerical computations indicates that the MASP associated to $\Sigma_{2}(s)$ is larger than $T_{2}^{*}>T_{2}=2$. The problems now simplifies to the computation of the gains $k_{1}$ and $f_{1}$ such that the closed loop system $\Sigma_{1}$ results in a strictly passive operator with $T^{*} \geq \min \{1,2\}=1$. This MASP is computed for a determined value of $P_{c}$.

The system $\Sigma_{1}(s)=\frac{1}{s+k_{1}}+f_{1}$ has a state space realization given by: $A_{c}=-k_{1}, B_{c}=1, C=1$ and $D=f_{1}$.

Note that the solution is not unique, and various solutions involving high-gain may be possible. In order to obtain moderate gains, some computations need to be done. Firstly, an energy storage function can be defined, i.e. considering $k_{1}, f_{1}>0$, we have

$$
W=\left(\begin{array}{ll}
-2 k_{1} p & p-1 \\
p-1 & -2 f_{1}
\end{array}\right) \leq 0
$$

and one may choose $p=1$. It is straightforward to compute

$$
W_{1}=\left(\begin{array}{cc}
2 k_{1}^{2} & -\frac{3}{2} k_{1} \\
-\frac{3}{2} k_{1} & 1
\end{array}\right)
$$

and the first order approximation of $T^{*}$ is

$$
T^{*}=\frac{-1}{\operatorname{tr}\left(W^{-1} W_{1}\right)}=\frac{2 f_{1}}{2 k_{1} f_{1}+1}
$$

Finally, the controller gains can be found solving this expression for $f_{1}$ with $T^{*}=1$. This gives the formula 
$f_{1}=0.5 /\left(1-k_{1}\right)>0$ and the valid range for the feedback gain is in an interval $0<k_{1}<1$. One possible choice is $k_{1}=1 / 2$, and $f_{1}=1$. This controller may satisfy the true MASP condition since the first order approximation of the MASP is conservative. Nevertheless, the exact value of $T^{*}$ can here be computed to ensure the correctness of this result, making $W_{d}(T)=0$ :

$6 e^{2\left(-\frac{1}{2} T\right)} T+4-8 e^{-\frac{1}{2} T}-2 T+4 e^{2\left(-\frac{1}{2} T\right)}-4 e^{-\frac{1}{2} T} T+T^{2}=0$

The true value of MASP is, as expected, $T^{*} \simeq 1.86>1$.

To illustrate the result, two simulations have been performed with $k_{1}=1 / 2$ fixed. They are shown in Figure 3 . The left one is obtained with $f_{1}=1$ and the result is stable. As discussed, lower values of $f_{1}$ do not guarantee stability. For example, $f_{1}=0.4$ produces an unstable closed loop system, as it is depicted in the right side of the figure.
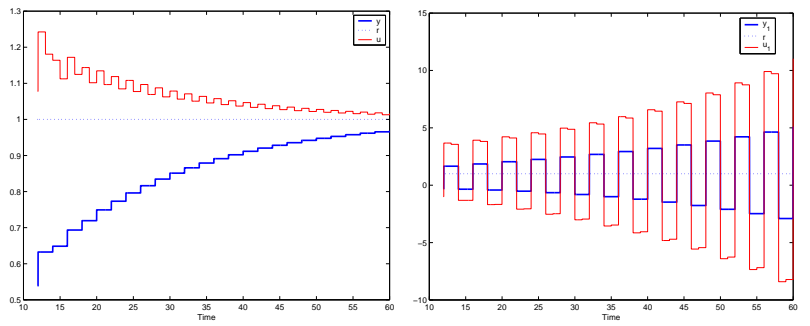

Fig. 3. Trajectories $y_{1}(k),-y_{2}(k)$ of the simulated example. Reference is $r=1$. Figure at left show a case where $f_{1}=1$ leading to a stable behaviour. The right figure shows the unstable case when $f_{1}$ does not satisfy the MASP condition, i.e. $f_{1}=0.4$.

As a matter of fact, another simulations with sampling times $T_{1}=T_{2} / 2=3$ have been repeated. The system with $f_{1}=1$ is stable whereas $f_{1}=2$ results in instability. Indeed, $f_{1}=1.64$ produces an oscillating system. A further investigation shows that the corresponding MASP for last value of $f_{1}$ is $T^{*} \simeq T_{1}=3$.

\section{CONCLUSIONS}

In this paper we have presented a constructive algorithm to design local controllers for feedback systems that are interconnected via time-varying and asynchronous sampling. The study was based on dissipation inequalities that results on the notions of (MASP) MAximum Sampling time preserving Passivity. This results is consistent with other works reported in the literature; Laila et al. [2001], and Rohrer and Nosrati [1981]. We have proposed a numerical method to compute the MASP for linear systems, and we did propose a numerical algorithm to compute local feedback loops providing a MASP compatible with the maximum sampling-time upper bound of each sub-system. The results was that if we interconnect feedback continuous-time systems having a suitable dissipation properties via asynchronous sampling, there exists upper bounds on these sampling-time preserving dissipation, such that the resulting inter-connection is stable. This is somewhat expected from the known results concerning passive continuous-time system interconnections. Finally, we propose a way to solve the "inverse" problem to the one mentioned above. That is, how to design local continuoustime feedback such that, given a maximum upper bound on the sampling time, the resulting MASP of each subsystem is consistent with this bound. Some simulation results were carried out to validate and to illustrate this design.

\section{REFERENCES}

M. Araki. Recent development in digital control theory. In Proc. of the $12^{\text {th }}$ IFAC World Congr., volume 9, pages 951-960, 1993.

B. Brogliato, R. Lozano, O. Egeland, and B. Maschke. Dissipative Systems Analysis and Control. Theory and Applications. Springer-Verlag, London, second edition, 2007.

L. El-Ghaoui and S.-I. Niculescu, editors. Advances in Linear Matrix Inequality Methods in Control. Advances in Design and Control. SIAM, Philadelphia, 2000.

D. Harville. Matrix Algebra from a Statiscian's Perspective. Springer-Verlag, New York, 1997.

A. Hassibi, S. Boyd, and J. How. Control of asynchronous dynamical systems with rate constraints on events. Stanford University, 1999.

P. Hokayem and M. Spong. Bilateral teleoperation: An historical survey. Automatica, December 2006.

D. Laila, D. Nesic, and A. Teel. Open and closed loop dissipation inequalities under sampling and controller emulation. April 2001.

H. J. Márquez. Nonlinear Control Systems: Analysis and Design. Wiley-Interscience, May 2003.

P. Marti, R. Villa, G. Fohler, and J. Fuertes. A discrete-time controller design method to tolerate non-equidistant sampling and actuation. Technical report, Automatic Control Department. Universidad Politécnica de Cataluña, January 2002. ESAII-RR-0205.

R.-M. Murray, K.-J. Astrom, S. Boyd, R. Brockett, and G. Stein. Future directions in control in an informationrich word. IEEE Control System magazine, April 2003.

E. Navarro-Lopez. Several dissipativity and passivity implications in the linear discrete time-setting. Mathematical Problems in Engineering, 6:599-616, 2005.

E. Navarro-López. Dissipativy and Passivity-related Properties in Nonlinear Discrete Time Systems. PhD thesis, Universidad Politécnica de Cataluña, 2002. Instituto de Organización y Control de Sistemas Industriales.

J. Nilsson and B. Bernhardsson. LQG control over a Markov communication network. Lund Institute of Technology, 1997.

A. Rantzer and C. Byrnes, editors. Directions in Mathematical Systems Theory and Optimization, chapter Systems with Lebesgue Sampling, pages 1-13. SpringerVerlag, Berlin, 2003.

D. Robert. Contribution à l'interaction commande/ordonnancement. $\mathrm{PhD}$ thesis, Institut National Polytechnique de Grenoble, Laboratoire d'Automatique de Grenoble - Ecole de l'Energie et du Traitement de l'Information, January 2007.

R. Rohrer and H. Nosrati. Passivity considerations in stability studies of numerical integration algorithms. IEEE Transactions on Circuits and Systems, CAS-28 (9):857-866, September 1981. 